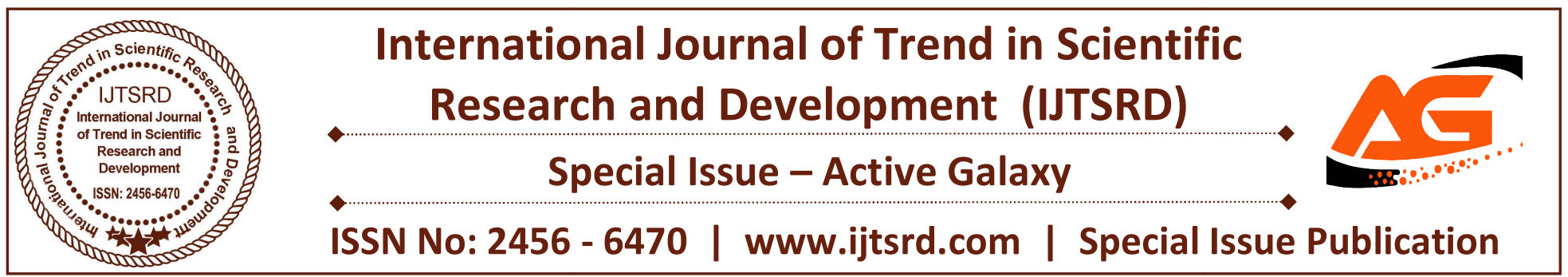

\title{
Vehicles Awake Routing Protocol with Analysis Determine Knowledge Perception for VANET
}

\author{
Mr. P. Senthil ${ }^{1}$, Mrs. A. Jayanthi ${ }^{2}$, R. Shobana $^{3}$ \\ ${ }^{1,2}$ Assistant Professor, ${ }^{3} \mathrm{PG}$ Scholar \\ ${ }^{1}$ Dept of Computer Science and Engineering, ${ }^{2}$ Dept of Information Technology, \\ ${ }^{3}$ Dept of Master of Computer Application \\ Veltech Hightech Dr Rangarajan Dr Sakunthala Engineering College \\ Avadi, Chennai, India
}

\section{ABSTRACT}

The Vehicular Ad-hoc Networks (VANET) ascents as an surface technology for smart transport as observed in the latter date decennary. The routing is the important element for keeping effectual communication between smart vehicles, which need to be entreated snappily. A traffic-aware routing protocol (TARCO) that considers integrated real-time traffic conditions for integrating delivery paths over a vehicular environs is presented. Routing in VANETs plays 0 crucial role in production of networks. VANET protocols are classified as topology based and position based concordat. Device-to-device (D2D) communication is admired as a propitious technique as granting the reliable integration between vehicles. The D2D-based vehicle-to-vehicle communication links coincide by recycling the similar sequence property, solution in a more intricate Combat scenario. Thus the access mode switch and resource allocation between cellular and VANETs become a challenging issue. Each road segment was then assigned a weight according to the overall view of the traffic conditions and updated systematically to reflect traffic variations. Finally, the road segments providing operative and dependable data paths were used to frame a routing path with latched connectivity and a short distribution lag to the destination. Simulation results showed that the use of TARCO leads to high network performance in terms of the packet delivery ratio, end-to-end delay and communication upward.

Keywords: VANET, TARCO, D2D.

\section{Introduction}

The vehicular ad hoc network (VANET) is an emanate paradigm that has the prepatent to improve the inviolability and efficiency of portage in the future. Since the propagation and migration pattern of vehicles are constrained by road layouts and traffic systematizations, digital maps and geographical locations of vehicles can be useful for making routing decisions. However, varying vehicle mobility often results in unreliable connectivity and inferable irrational service quality. Therefore, routing protocols for VANET should be capable of overcoming these drawbacks.

Position-based routing protocols are the most appropriate for VANET. In these protocols, genuine network and traffic information are essential to avoid the selection of unfavorable paths. Some studies have affiliate the concept of dividing a road segment into fixed-size cells and then selecting a vehicle closest to the cell centre as the traffic information representative in each cell.

However, the distribution of vehicles is often random, and some of the selected information collectors may not always be located close to the corresponding cell centres. Consequently, some vehicles are not included in the information collection process because of the limited hauling inclusion of the selected information collectors. Hence, the collected information may not represent the real road conditions. To address the aforementioned problem, a cooperative information 
collection method was developed in this study. This method dynamically selects the most relevant vehicular nodes at links as information aggregators and nodes on road segments as information collectors to convergence collect hierarchical information. The hierarchical information collection scheme employed provides a discursive view of the traffic to a trafficaware routing protocol called TARCO. This protocol uses information on real-time vehicular traffic and data network traffic for performing lightweight road enumerating and assembling a conductive, dependable and flexible data route to the intention. The enumerate mechanism estimate the route substantiality over each road according to the collected traffic information, and assigns a weight to it. All weights are systematically updated to resonate divergence inroad traffic. The data route systematization mechanism then recognize a routing path with guaranteed connectivity and a short delivery delay to the destination according to the scoring results.

\section{Literature survey}

2.1 Title: Traffic-aware routing protocol with cooperative coverage-oriented information collection method for VANET, "Chun-ChihLo, Yau-Hwang Kuo" (2017)

Description: This protocol dynamically selects the information aggregator of a junction from the nodes located at the junction. The node with relatively stable mobility and the longest staying-in-junction time is chosen as the information aggregator. The CIADP consists of two phases: the successor selection phase. The proposed system appraisal could be done by In Junction-Based Traffic-Aware Routing (JTAR), both the vehicular density and data traffic load are considered for determining a reliability-oriented score for each junction. With such consideration and the location of nodes helps the delivery of data packets through routes with high vehicular density and connectivity.

2.2 Title: Intersection-based Distance and TrafficAware Routing (IDTAR) Protocol for Smart Vehicular Communication, Abdelmuttlib Ibrahim AbdallaAhmed (2017)

Description: The main aim of Inter-vehicle ad-hoc network and introduced Intersection-based Distance and Traffic-Aware Routing (IDTAR) protocol. Many factors affect the communication between the vehicles such as topology fragmentation which results in the phenomenon of Local-Maximum-Problem that lead to packet delivery failure. The traditional routing schemes failed to address the problem of the trade-off between Packet-Delivery-Ratio and the high cost, in term of End-to-End-Delay, of packet recovery from the failures that occurs frequently because of LocalMaximum-Problem.

Sivarajet al. propose a cluster head election mechanism to effectively manage VANET subclusters, which guarantees multimedia communication services over spatially-apart vehicular groups a cluster head election mechanism to effectively manage VANET sub-clusters, which guarantees multimedia communication services over spatially-apart vehicular groups. A cloud-assisted message downlink dissemination scheme is investigated where safety messages in the cloud server are delivered to the suitable mobile gateways and then the messages are disseminated among neighboring vehicles via V2V communication. We propose an optimal access mode selection and resource allocation scheme in a cellularVANET heterogeneous network, where vehicular users make decisions to select an appropriate mode and obtain resources between cellular communication and D2D-based V2V communication; meanwhile CUEs select their own best serving BS. A user aggregate utility maximization problem is formulated with the joint consideration of network topology, transmission delay for D2D-based V2Vcommunication and power reduction, which can serve as a benchmark for other scenarios, such as mode switching for more complicated network structures.

2.3 Title: Vehicular Networking: A Survey and Tutorial on Requirements, Architectures, Challenges, Standards and Solutions, Georgios Karagiannis, OnurAltintas, EylemEkici, Geert Heijenk, BoangoatJarupan, Kenneth Lin, and Timothy Weil, (2011)

Description: The main advantages are Intersection collision warning: in this use case, the risk of lateral collisions for vehicles that are approaching road intersections is detected by vehicles or road side units.

Lane change assistance: the risk of lateral collisions for vehicles that are accomplishing a lane change with blind spot for trucks is reduced.

Co-operative merging assistance: vehicles involved in a junction merging maneuver negotiate. 
Intersection collision warning: in this use case, the risk of lateral collisions for vehicles that are approaching road intersections is detected by vehicles or road side units.

Raya et al. extensively discussed that vehicles might become fake or their reliabilities become partially or fully compromised by attackers, which require their reliabilities to be revoked. They proposed a datacentric trust model that computes trust in each individual piece of data.

2.4 Title: A Secure Trust Model Based on Fuzzy Logic in Vehicular Ad Hoc Networks With Fog Computing, Seyed Ahmad Soleymani, Abdul Hanan Abdullah, Mahdi Zareei, Mohammad Hossein Anisi,Cesar Vargas-Roales, Muhammad Khurram Khan, Shidrokh Goudarzi (2017)

Description: In the proposed model, we consider a module to assess authentication of a sender as the rest and main requirement for any security system. $\bar{C}$ ertain data associated to the transmitting

node are extremely essential in VANET. Such data can be identi_cation information of the senders in addition to their features and locations. A decisionmaking module decides on the sender of the event message. The receiver accepts and relays on the event message if the sender is trustable, denies it otherwise.

2.5 Title: Comparative Study of VANET Routing Protocol, Qi-wu Wu*, Wen Wen, QingziLiu (2014)

Description: On-demand Routing does not need to update Routing table, just need to update accord to the Routing algorithm established by agreement for Routing when node have communication needs, a typical on-demand Routing protocols includes the Dynamic Source Routing. So the structure of the cluster often changes, each time to establish cluster structure and it will take the corresponding resource and cause some influence for time delay, if it will be used in car networking, we need to more carefully choose clustering method.

2.6 Title: Non DTN Geographic Routing Protocols for Vehicular Ad Hoc Networks, Ramin Karim, NorafidaIthnin, ShukorAbdRazak, SaraNajafzade (2011)

Description: A-STAR-Anchor based Street and Traffic Aware Routing This routing follows the approach of anchor-based routing with street awareness. This is having consciousness of the physical environment around the vehicles; the use of anchor-based routing is not novel either. It consists of including within the packet header the list of junctions (anchors) that the packet must traverse. This approach has been employed in the GSR protocol. In fact, ASTAR relies on GSR to perform the routing task.

2.7 Title: Performance Improvement of Cluster-Based Routing Protocol in VANET, MICHEL KADOCH, AHMAD ABUASHOUR (2017)

Description: Geographic-based routing protocols or Location-based routing protocols combine the position information with topological knowledge of the actual road map and surroundings.CBR protocols are widely used to improve the scalability of VANET environment and to reduce the control overhead message. Although the clustering techniques are minimizing the routing control overhead, frequent $\mathrm{CH}$ elections increase the control overhead associated with the re-election process.In geographic-based routing protocols, the data is transmitted directly from the source to the destination without initiating any route discovery process.

\section{Related Work:}

Implementation is the stage of the project when the theoretical design is turned out into a working system. Thus it can be considered to be the most critical stage in achieving a successful new system and in giving the user, confidence that the new system will work and be effective.

The implementation stage involves careful planning, investigation of the existing system and it's constraints on implementation, designing of methods to achieve changeover and evaluation of changeover methods.

\subsection{Collaborative traffic information collection}

CTIC scenario where the coverage gap, absence of traffic, and traffic variation may severely affect the CTIC performance. The coverage gap indicates that the overall coverage area of road segments by information collectors is insufficient for collecting accurate traffic information.

TARCO was developed to achieve three objectives: sufficient data collection coverage, a high data return ratio and robust data collection. Achieving the first 
objective requires the selection of an appropriate number of information collectors (NIC) to maximize the total information collection coverage. The second objective was set to ensure that the collected information can be sent to an appropriate information aggregator. Achieving the third objective requires the use of a data collection mechanism that is efficient and adaptable to highly dynamic environments.

\section{ATDq $=\mathrm{NICi} \times$ ATDiq $+\mathrm{NICi}{ }^{\prime} \times$ ATDiqNICi + NICi (1)}

\section{$\mathrm{ACLq}=\max$ CLiq, $\mathrm{CLiq}(2)$}

Whenever an information aggregator receives a TIC packet, it updates the weight of the corresponding road segment according to the information carried by the TIC packet and reports the new weight to the location service.

\section{$\mathrm{Wq}=\mathrm{a}$ ATDq $/ \mathrm{TDmax} \times(1-\mathrm{a}) \mathrm{ACLq} / \mathrm{CWmax}$}

Ta is given by $2 \mathrm{RTTkr} / \mathrm{p}$, and Tc is determined as TTLJk/3p, where $p$ is the timers' countdown duration, RTTkr is the average round trip time in the transmission range of $\mathrm{k}$, and TTLJk is estimated from $\mathrm{k}$ 's current velocity and the remaining distance for $\mathrm{k}$ to leave the junction. In other words, the new aggregator contention phase favours the node that remains at a junction for the longest period of time. Because of the highly dynamic nature of VANET, nodes continue to enter and leave a junction, leading to extra overhead for replacing the current information aggregator. The proposed two-phase protocol can alleviate this problem because of the cooperation between the current aggregator and newly arriving nodes.

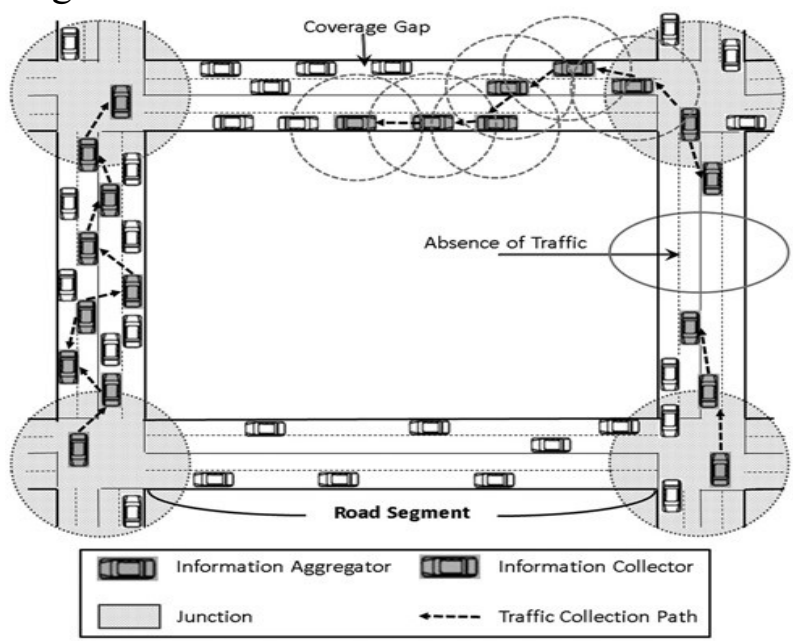

Fig. 1 Cooperative TIC scenario

\subsection{Frivolous real-time road scoring}

This protocol is used for constructing a data delivery path with the lowest congestion probability and the highest stability in terms of connectivity. The balance of network traffic load over the roadway is also considered. The ADRC protocol consists of the intraand inter-segment phases and executes the following protocol. Initialization: A source node $\mathrm{S}$ on road segment RSS initiates a data delivery request to the destination node $\mathrm{D}$ on road segment RSD.

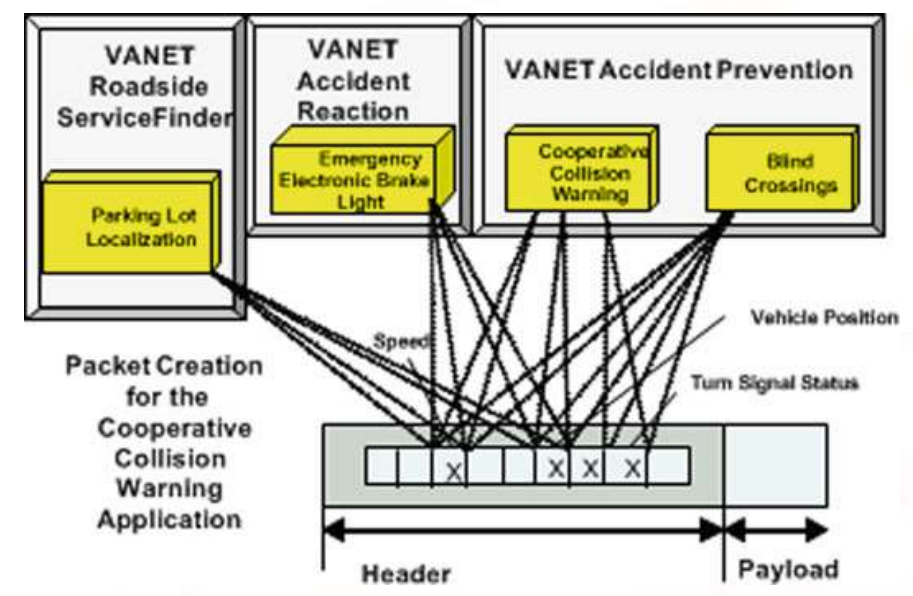

Fig 2.Architecture of vanet

\subsection{Tractable data route construction}

$\mathrm{S}$ obtains the position of $\mathrm{D}$ from the location service. $\mathrm{S}$ executes the Dijkstra-oriented algorithm to formulate an inter-segment data delivery plan (IDDP) for conveying data packets from S to D; subsequently, $\mathrm{S}$ becomes the current data forwarder. The current data forwarder searches for its successor along the road segment for delivering the given data packet towards an adjacent junction in the IDDP.

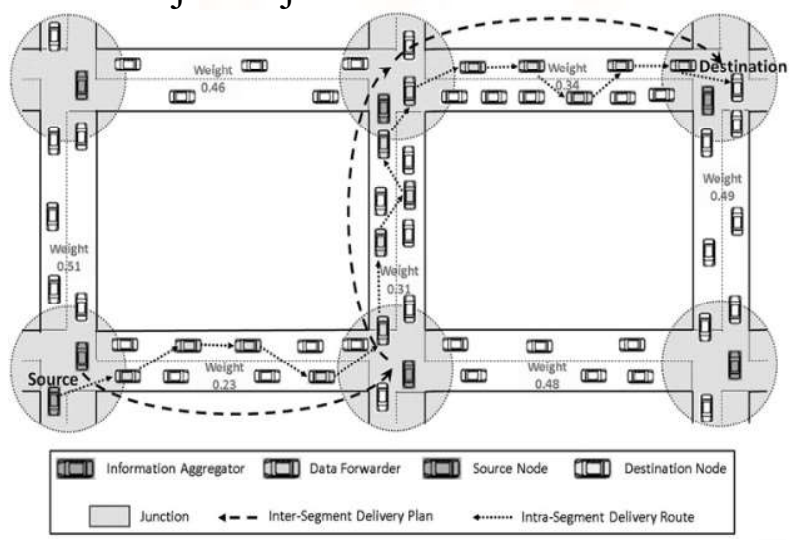

Fig.3Operational scenario of the ADRC protocol 
When the CCIPP is executed, a probe packet called TIC packet is delivered to information collectors for collecting data from a road segment. Where the traffic density field records the traffic density along a given road segment, the channel load field records the workload of the data delivery channel over the road segment and the timestamp field records the timestamp of the information collection cycle in which the TIC packet is produced. The traffic density field has two attributes: NIC and average traffic density (ATD).

NIC represents the NIC that have contributed traffic information to the TIC packet, and ATD denotes the average density of nodes along a given road segment. The channel load field represents the workload in the data delivery channel over a given road segment. In each information collection cycle, two TIC packets are delivered along a given road segment between junctions $\mathrm{j} 1$ and $\mathrm{j} 2: \mathrm{TICj} 1 \mathrm{j} 2$ is delivered from $\mathrm{j} 1$ to $\mathrm{j} 2$ and $\mathrm{TICj} 2 \mathrm{j} 1$ is delivered from $\mathrm{j} 2$ to $\mathrm{j} 1$.

\section{Simulation Result}

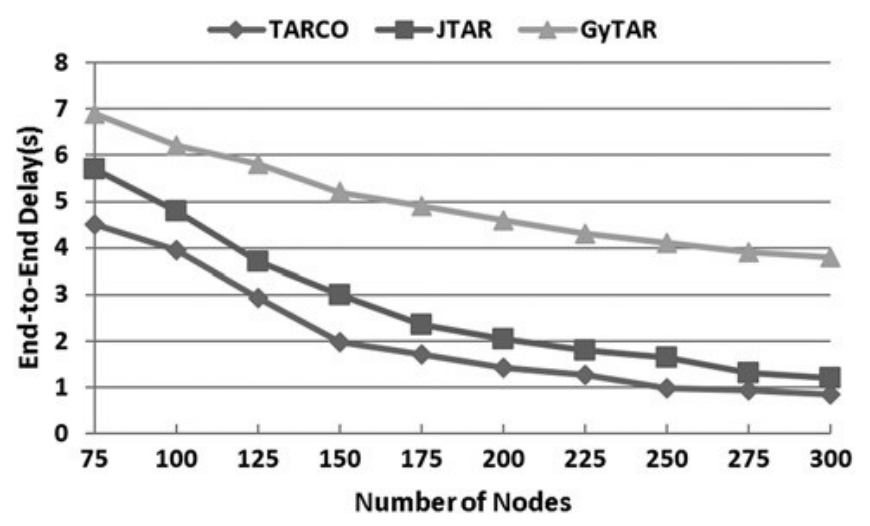

Fig.4 Performance comparison in terms of the endto end delay

TARCO has the shortest end-to-end delay among the protocols compared. GyTAR considers high vehicular traffic density on a road segment as a positive condition. However, high vehicular density may cause network congestion and degrade the network performance. TARCO and JTAR both consider the network traffic load for determining the routing path. Therefore, alternative connected road segments with less vehicular traffic density and less network traffic load are also considered for packet delivery.

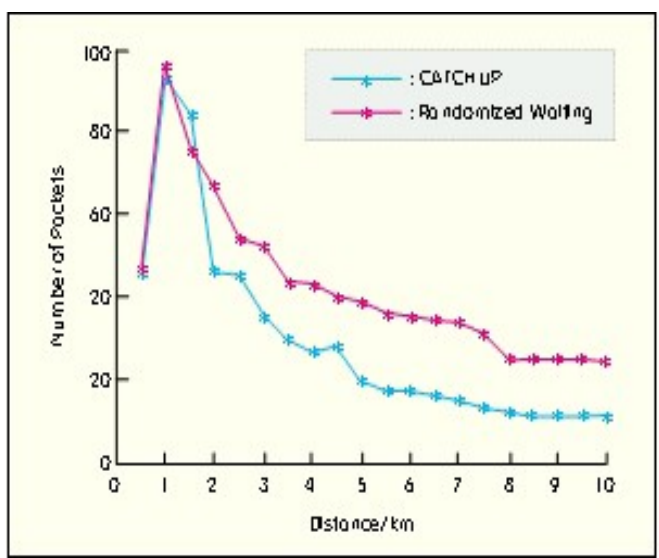

Fig.5 Performance of in network data aggregation

Furthermore, although both JTAR and TARCO allow the routing path to be recalculated dynamically during data transmission, in TARCO, information aggregators obtain a more accurate view of the road conditions. Therefore, TARCO is more responsive to variasssstions in the network. In addition, the probe packet merging used in JTAR and TARCO helps information aggregators acquire traffic information faster.

\section{Future Enhancement}

The selection is based on real-time traffic density, the traffic load of the corresponding road segment, and the distance to the destination. To evaluate the performance of CORA protocol, we

Implemented a bidirectional highway scenario. we have proved that the IDVR protocol outperforms VDLA, IRTIV, and GPCR in terms of end-to-end delay and throughput.

\section{Conclusions}

In this study, TACBRCO is proposed to improve routing performance in urban vehicular environments. The improvement is achieved by using a cooperative information collection method that provides highly accurate information on road traffic and data network conditions. This accurate traffic information renders the weighting of road segments highly reliable.

Accordingly, TACBRCO can generate highly efficient routing paths and adjust the path dynamically to respond to variations in the road traffic and data channel load. TACBRCO also applies a probe packet merging scheme, which not only shortens the time spent for collecting traffic information but also reduces the communication overhead required for collecting the information. 


\section{References}

1. Chun-Chih Lo, Yau-Hwang Kuo," Traffic-aware routing protocol with cooperative coverageoriented information collection method for VANET" (2017).

2. Abdelmuttlib Ibrahim Abdalla Ahmed, "Intersection-based Distance and Traffic-Aware Routing (IDTAR) Protocol for Smart Vehicular Communication" (2017).

3. Yanjia Qi, Hongyu Wang1, Lingyan Zhang, Bing Wang," Yanjia Qi1, HongyuWang1, Lingyan Zhang1, Bing Wang2”.

4. Hong Zhong, Jingyu Wen, Jie Cui_, and Shun Zhang," Efficient Conditional Privacy-Preserving and Authentication Scheme for Secure Service Provision in VANET" (2016).

5. MuathObaidar, M. AliI, IhsanShahwanI, SuhaibObeidar, AndiToce, "DYNAMIC SUPPRESSION BROADCAST SCHEME FORVEHICLE ADHOC NETWROKS (VANET)".

6. Qi-wu Wu*, Wen Wen, Qingzi Liu, "Comparative Study of VANET Routing Protocol” (2014).

7. ZinebSqualliHoussaini, "Comparative Study of Routing Protocols Performance for Vehicular Adhoc Networks" (2017).

8. Ramin Karim, NorafidaIthnin, ShukorAbdRazak, SaraNajafzade," Non DTN Geographic Routing Protocols for Vehicular Ad Hoc Networks" (2011).

9. XiaoyuDuan, Yanan Liu, and Xianbin Wang,"SDN Enabled 5G-VANET: Adaptive Vehicle Clustering and Beam formed Transmission for Aggregated Traffic" (2017).

10. Ahmad Abuashour and Michel Kadoch,'Performance Improvement of ClusterBased Routing Protocol in VANET" (2017).

11. Seyed Ahmad Soleymani, Abdul Hanan Abdullah, Mahdi Zareei, Mohammad Hossein Anisi,Cesar Vargas-Roales, Muhammad Khurram Khan, Shidrokh Goudarzi ,
"A Secure Trust Model Based on Fuzzy Logic in Vehicular Ad Hoc Networks with Fog Computing" (2017).

12. GeorgiosKaragiannis, OnurAltintas, EylemEkici, Geert Heijenk, BoangoatJarupan, Kenneth Lin, and Timothy Weil,

"Vehicular Networking: A Survey and Tutorial on Requirements, Architectures, Challenges, Standards and Solutions", IEEE Communications Surveys \& Tutorials.(2011)

13. H. B. Qin ? Z. Y. Xiao. "Stable clustering routing Protocol based on location information", Journal of South China University of technology. 2010. 38 (6). 2010: 242-252.

14. M. Mohammed, K. K. Xu? W. W. Xia et al. "Desert scene used car networking and clustering routing algorithm", Journal of communication, 2012, 33(10): 166-174.

15. [15]. J. Song, L. L. Yang, J. L. Sun, et al. "Under the environment of urban road vehicle ad-hoc network clustering routing mechanism", Journal of Chongqing Jiaotong university (natural science edition), 2013, 32(1): 108-135.

16. S. Biswas, R. Morris, "Opportunistic routing in multihop wireless networks." In: Proc. Of the $A C M$ SIGCOMM 2005. New York: ACM Press, 2005: 133-143.

17. J. Burgess, B. Gallagher, D. Jensen, et al. "MaxProp: Routing for Vehicle-Based DisruptionTolerant Networks", In Proc. Of 25th IEEE Intenational Conference on Computer Communications (INFOCOM). 2006.1-11.

18. V. Nauov, T. R. Gross, "Connectivity-aware routing(CAR) in vehicular ad-hoc networks", 2007.1919-1927.

19. Z. Jing, H. C. Guo, VADD: "Vehicle-Assisted Data Delivery in Vehicular Ad Hoc Networks", IEEE Transaction on Vehicular Technology. 2006, 57(3):1910-1922?

20. K. C. Lee, U. Lee, et al. "TO-GO: Topology-assist geoopportunistic Routing in urban vehicular grids", Wireless On-Demand Network Systems and Services, 2009. 